Measurement of the body force field of plasma actuators

This article has been downloaded from IOPscience. Please scroll down to see the full text article.

2011 J. Phys. D: Appl. Phys. 44045204

(http://iopscience.iop.org/0022-3727/44/4/045204)

View the table of contents for this issue, or go to the journal homepage for more

Download details:

IP Address: 131.180.130.109

The article was downloaded on 10/05/2012 at $14: 16$

Please note that terms and conditions apply. 


\title{
Measurement of the body force field of plasma actuators
}

\author{
M Kotsonis, S Ghaemi, L Veldhuis and F Scarano \\ Delft University of Technology, Kluyverweg 1, The Netherlands \\ E-mail: m.kotsonis@tudelft.nl \\ Received 12 October 2010, in final form 18 November 2010 \\ Published 6 January 2011 \\ Online at stacks.iop.org/JPhysD/44/045204
}

\begin{abstract}
A novel technique is proposed and investigated for the estimation of the body force field resulting from the operation of a dielectric barrier discharge plasma actuator. The technique relies on the measurement of the spatio-temporal evolution of the induced velocity field using high-speed particle image velocimetry (PIV). The technique has the advantage of providing spatial distribution of the body force vector field. A full Navier-Stokes term decomposition is applied on the evolving field along with additional closure norms in order to decouple the pressure gradient and body force terms. Results are compared with load-cell measurements of the direct reaction force and also momentum balance calculations based on the PIV field. Agreement between the different methods is observed. The data can easily be incorporated in computational flow solvers and also be used for validation and calibration of numerical plasma models.
\end{abstract}

(Some figures in this article are in colour only in the electronic version)

\section{Introduction}

Plasma actuators have been studied extensively in recent years, as flow control devices. Their low power consumption, lack of moving parts and robustness render them ideal for flow manipulation. Plasma actuators have been used in several studies aiming at separation control [1], turbulent drag reduction [2], boundary layer control [3-5], noise reduction [6] and laminar-turbulent transition delay [7]. Comprehensive reviews on plasma actuators for aerodynamic flow control have been published recently $[8,9]$.

There are several implementations of the actuators, the most popular being the dielectric barrier discharge (DBD) (figure 1). These are based on the ionization of air using ac high voltage (HV). In the most conventional configuration, two electrodes are employed. The electrodes are separated by a dielectric layer which prohibits arc forming and allows accumulation of the ionized gas in the vicinity of the exposed electrode. The exact mechanisms of interaction between the weakly ionized gas and neutral air are still debated. It is, nevertheless, commonly accepted that collisional processes between the heavy plasma species (mostly ions) and neutral air is responsible for the momentum transfer [10]. At a macroscopic scale the model of an exerted body force on the fluid seems to describe the effect with reasonable accuracy.

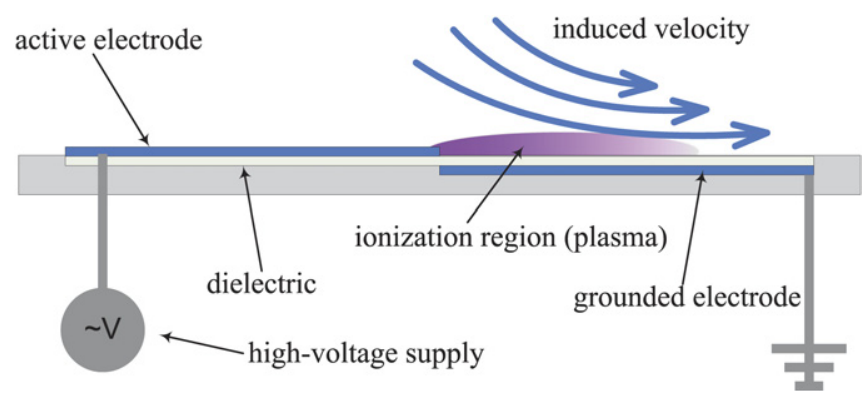

Figure 1. Geometrical configuration and operation of the plasma actuator.

A large amount of simulation studies have been conducted in order to simulate the underlying physics of the ionization process [11-13]. These vary in model complexity, from simple phenomenological models to first-principles fluid models [14]. Extended simulations for multi-species fluids have also been investigated [15]. In the majority of these modelling approaches the final goal is to determine the exerted body force on the fluid. A number of studies on flow solvers implementing plasma actuators have successfully coupled the effect of the actuator with the flow dynamics $[16,17]$. The capabilities of the actuators suggest the feasibility of their implementation in several flow control scenarios. It is, therefore, desirable to have an efficient and accurate model of the actuator effect. 
The experimental investigation on the actuator's operation has also received substantial attention. Several studies focus on the investigation of the induced velocity field [18-20] and the produced thrust from the actuators [21-23]. These methods are robust and fast but they present some drawbacks which render the results difficult to be used as an input in flow solvers, an area that so far has been served only by numerical plasma modelling studies. An inherent disadvantage is that a part of the measured thrust does not correspond to the actuator effect but to the shear forces developed between the flow and the surface. Another shortcoming is also their inability to provide any information on the spatial distribution of the force field, information that is vital in cases of discrete, unsteady and localized actuation essential to the majority of active closedloop flow control concepts. To the authors' knowledge no experimental investigation on the determination of both the magnitude and the spatial distribution of the body force vector field has been published to date.

This paper aims at the development of a novel experimental approach for determining the amplitude and spatial distribution of the body force exerted on the flow by a plasma actuator. The proposed method will be evaluated against well-established thrust measurement techniques used as a benchmark. A description of the methodology for the new body force method as well as the benchmark thrust measurement techniques will be given in the following section. A description of the experimental setup and a discussion on the results are finally provided.

\section{Methodology}

The determination of the induced body force can be done experimentally in several ways. Due to the nature of the momentum transfer mechanisms direct measurements of the ionized species density in the plasma region can only be obtained using molecular level diagnostics. The species density can be used to derive the Coulomb forces exerted by the electric field. Taking into account the complexity of such an approach, only indirect methods based on the induced flow field or reaction forces can be feasibly employed in the time span such an experiment allows.

In this study two techniques are applied to determine thrust produced by the operation of the actuator. These will be used as benchmark cases for validating and verifying the developed force estimation technique. More specifically, the techniques employed are direct thrust measurements using a highly sensitive load cell, and thrust calculation via momentum balance of the induced flow field.

\subsection{Body force estimation}

The proposed body force measurement technique determines the spatial distribution of the plasma-induced body force from time-resolved velocity field. The required information is obtained using a high-speed PIV system as it is shown in a subsequent section of this paper. An analysis of the velocity field using the full Navier-Stokes (NS) equations can provide the body force. The 2D incompressible NS equations in the presence of body forces read as

$$
\frac{\partial \boldsymbol{U}}{\partial t}+\boldsymbol{U} \cdot \boldsymbol{\nabla} \boldsymbol{U}-v \boldsymbol{\nabla}^{2} \boldsymbol{U}=-\boldsymbol{\nabla} p+\frac{\boldsymbol{F}}{\rho}
$$

where $\boldsymbol{U}$ is the 2D velocity field, $p$ is the static pressure, $v$ is the kinematic viscosity of the fluid and $\rho$ is the density.

A first approach in deriving the body force $(\boldsymbol{F})$ is to apply (1) only for the first moments after the actuation. During this stage the flow is expected to accelerate only around the region of the exerted body force where the rest of the field is still relatively quiescent. For initial conditions of zero velocity the convective, viscous and pressure gradient terms are assumed to be relatively small and can be neglected. This reduces (1) to

$$
\frac{\partial \boldsymbol{U}}{\partial t}=\frac{\boldsymbol{F}}{\rho}
$$

which is basically Newton's second law. This method will be referenced as the 'reduced method' throughout this paper.

In order to use the full NS equations to derive the force it is necessary to calculate all the terms involved. The acceleration, convective and viscous terms are obtained from the available spatio-temporal data of the velocity field. In the absence of body force terms the only unknown is the pressure gradient which can be obtained using (1) from the measured velocity field. In the case of the plasma actuator, the body force term appears as one extra unknown. Physically, the pressure gradient term and the body force term are equivalent and one can be used to explain the phenomena related to the other. Nevertheless, the expression of the plasma effect through a volume distributed body force is a convenient way of imposing the effect in a flow solver. To be able to bypass the problem of one extra unknown, two major assumptions must be made: (1) the body force remains quasisteady over a large number of HV cycles and (2) the pressure gradient prior to the actuation is zero. The assumption for a quasisteady force is justified by taking into consideration the difference in time scales involved between one HV cycle and the acceleration of the flow. As mentioned by Enloe et al [18], the body force production is not constant within one HV cycle. Nevertheless, the high frequency of operation (order of $\mathrm{kHz}$ ) and the hydrodynamic 'inertia' of the flow reduce the effect to quasisteady in time. With these assumptions, (1) is differentiated in time:

$$
\frac{\partial^{2} \boldsymbol{U}}{\partial t^{2}}+\frac{\partial(\boldsymbol{U} \cdot \boldsymbol{\nabla} \boldsymbol{U})}{\partial t}-v \frac{\partial\left(\boldsymbol{\nabla}^{2} \boldsymbol{U}\right)}{\partial t}=-\frac{\partial(\boldsymbol{\nabla} p)}{\partial t} .
$$

The first assumption states that the body force is steady in time and its time derivative is reduced to zero. Equation (3) now involves only one unknown which is the time derivative of the pressure gradient. Integrating (3) back in time the pressure gradient can be calculated from:

$\int_{0}^{t}\left(\frac{\partial^{2} \boldsymbol{U}}{\partial t^{2}}+\frac{\partial(\boldsymbol{U} \cdot \boldsymbol{\nabla} \boldsymbol{U})}{\partial t}-v \frac{\partial\left(\boldsymbol{\nabla}^{2} \boldsymbol{U}\right)}{\partial t}\right) \mathrm{d} t=-(\nabla p)+A$

In the process of integration a constant $A$ appears which has to be defined. At this point the separation between the 


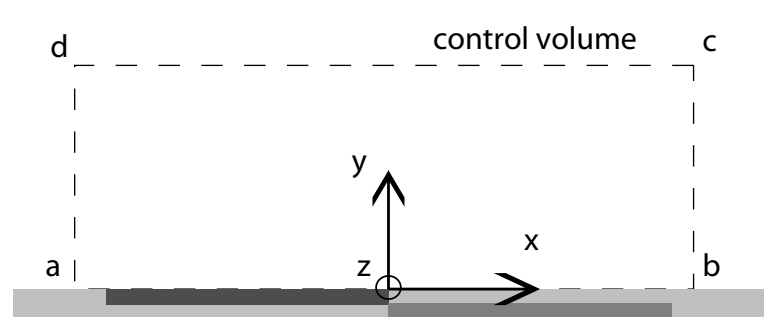

Figure 2. The control volume for the momentum balance equations.

pressure gradient due to the flow and the plasma body force is established. Based on the second assumption $\left(\left.(\nabla p)\right|_{t=0}=0\right)$ the integration constant is set to zero. Equation (4) is plugged back into (1) and leaves the body force term as the only unknown. Since the time gradient of the NS is used, this method will be referenced as the 'gradient method' throughout this paper.

In contrast to the two benchmark techniques the reduced and gradient methods have the advantage of providing spatial distribution of body force vectors instead of an integrated thrust value. The results from such analysis can be valuable for validation of numerical models of plasma actuators as well as an input into flow solvers investigating flow control concepts.

\subsection{Direct thrust measurement}

Direct thrust measurement is a straightforward technique which involves attaching the actuator to a load cell and measuring the force exerted by the induced flow on the actuator. By means of Newton's third law this force is equal and opposite to the total force the actuator exerts on the flow. The value of thrust can be valuable for parametric and optimization studies aiming at improving the actuator. Nevertheless, further clarifications must be made on what exactly is being measured. It has been reported repeatedly and also verified in this work that the induced flow field from the continuous operation of the actuator resembles a typical wall jet. As such, shear forces developing between the wall surface of the actuator and the accelerating fluid are also included in the load-cell measurement. Another issue with this technique and in general with integral techniques is the lack of spatial information.

\subsection{Thrust calculation from velocity measurements}

The second benchmark technique is based on measurements of the velocity field in the vicinity of the plasma actuator, obtained by PIV. It involves the definition of a control volume in the flow where the momentum flux is calculated at the boundaries of the domain and equilibrium with the internal forces is assumed (figure 2) ${ }^{1}$. This approach is well established in experimental and numerical investigations for the calculation of aerodynamic drag of airfoils and wings [24]. In a recent experimental study [23], it was used for estimating the thrust of plasma actuators. The calculated thrust includes both the body force from the actuator and also the friction force

1 For all subsequent figures and references to spatial dimensions and distances, the displayed coordinate system is used. The origin lies at the downstream end of the exposed electrode and on the flat surface. at the wall. This technique also suffers from lack of any information on the spatial distribution of the body force. More specifically applying the momentum balance equation on the control volume gives

$$
\boldsymbol{T}=\oint_{a b c d}(\rho \boldsymbol{U} \cdot \mathrm{d} S) \boldsymbol{U}+\oint_{a b c d} p \mathrm{~d} S
$$

where $\boldsymbol{T}$ is the integrated force exerted on the fluid. Equation (5) is developed for the boundaries taking account of the no-slip condition at the wall. This implies that the momentum flux across the wall is zero. Note that the boundaries where the unit vector perpendicular to the surface $(\mathrm{d} S)$ is negative take a minus sign:

- $x$-direction

$$
\begin{aligned}
T_{x}= & \rho \int_{d a} u_{x}^{2} \mathrm{~d} y+\rho \int_{c d} u_{x} u_{y} \mathrm{~d} x-\rho \int_{b c} u_{x}^{2} \mathrm{~d} y \\
& +\int_{d a} p \mathrm{~d} y-\int_{b c} p \mathrm{~d} y
\end{aligned}
$$

- $y$-direction

$$
\begin{aligned}
T_{y}= & \rho \int_{d a} u_{x} u_{y} \mathrm{~d} y+\rho \int_{c d} u_{y}{ }^{2} \mathrm{~d} x-\rho \int_{b c} u_{x} u_{y} \mathrm{~d} y \\
& +\int_{c d} p \mathrm{~d} x-\int_{b a} p \mathrm{~d} x .
\end{aligned}
$$

Due to the fact that pressure is not readily available from velocity data, a further assumption needs to be made. This states that if the control volume boundaries are far enough from the bulk of the plasma body force (near the inner electrode edge) pressure can be considered uniform and equal. A similar assumption is typically made in the case of airfoil drag estimation. It should also be noted here that for the $x$-direction the calculated force $\left(T_{x}\right)$ contains not only the contribution of the plasma actuator $\left(T_{x}^{\mathrm{p}}\right)$ but also the shear force between the flow and the surface $\left(T_{x}^{\mathrm{s}}\right)$. These assumptions reduce (6) and (7) to

$$
\begin{aligned}
& \text { - } x \text {-direction } \\
& T_{x}^{\mathrm{p}}+T_{x}^{\mathrm{s}}=\rho \int_{d a} u_{x}{ }^{2} \mathrm{~d} y+\rho \int_{c d} u_{x} u_{y} \mathrm{~d} x-\rho \int_{b c} u_{x}{ }^{2} \mathrm{~d} y
\end{aligned}
$$

- $y$-direction

$$
T_{y}=\rho \int_{d a} u_{x} u_{y} \mathrm{~d} y+\rho \int_{c d} u_{y}{ }^{2} \mathrm{~d} x-\rho \int_{b c} u_{x} u_{y} \mathrm{~d} y .
$$

\section{Experimental setup}

\subsection{The actuator}

In this investigation DBD actuators are fabricated using thin rectangular copper electrodes made out of self-adhesive copper tape (figure 1). The electrodes are $10 \mathrm{~mm}$ in length ( $x$-direction), with zero horizontal gap and $60 \mu \mathrm{m}$ of thickness. Their effective spanwise length (z-direction) is $300 \mathrm{~mm}$ for the load-cell measurements and $200 \mathrm{~mm}$ for the PIV measurements. The electrodes are separated by two dielectric layers of polyimide Kapton tape. The thickness of each layer 
Table 1. Test cases for thrust and body force investigation.

\begin{tabular}{lcl}
\hline Parameter & Value & $\begin{array}{l}\text { Base } \\
\text { value }\end{array}$ \\
\hline Upper electrode length $\left(l_{\mathrm{u}}\right)$ & $10 \mathrm{~mm}$ & - \\
Lower electrode length $\left(l_{1}\right)$ & $10 \mathrm{~mm}$ & - \\
Dielectric thickness $\left(t_{\mathrm{d}}\right)$ & $110 \mu \mathrm{m}$ & - \\
Horizontal gap $(\mathrm{g})$ & $0 \mathrm{~mm}$ & - \\
& Load-cell measurements & $8,9,10,11 \mathrm{kV}$ pp \\
Applied voltage $\left(V_{\text {app }}\right)$ & 8 to $14 \mathrm{kV}$ pp $($ steps of $0.5 \mathrm{kV})$ & $1,1.5,2 \mathrm{kHz}$ \\
Frequency $(f)$ & 0.5 to $4 \mathrm{kHz}($ steps of $0.25 \mathrm{kHz})$ & \\
& PIV measurements & $10 \mathrm{kV}$ pp \\
Applied voltage $\left(V_{\mathrm{app}}\right)$ & 8 to $16 \mathrm{kV}$ pp $($ steps of $2 \mathrm{kV})$ & $2 \mathrm{kHz}$ \\
Frequency $(f)$ & 1 to $4 \mathrm{kHz}($ steps of $1 \mathrm{kHz})$ & \\
\hline
\end{tabular}

is $50.8 \mu \mathrm{m}$. The total thickness of the dielectric, including adhesive, is approximately $110 \mu \mathrm{m}$. The upper electrode is connected to the HV output cable of a TREK 20/20C $\mathrm{HV}$ amplifier $\left(20 \mathrm{kV}_{\mathrm{pp}}, 20 \mathrm{~mA}\right)$ while the lower electrode is grounded.

The actuator operation is controlled remotely via a computer workstation where the driving signal is created in LabView software and is sent to the amplifier via a digital/analogue (D/A) converter. The amplifier provides direct readings of the output voltage and current through internal measurement probes. While the internal voltage probe gives sufficiently accurate readings the internal current probe has been found to be too slow to resolve high-frequency current fluctuations. This is typically the case with discharge currents occurring during plasma actuators operation. To resolve this, a resistance is placed between the lower electrode and the grounding cable and voltage is measured across it indicating the discharge current.

To reduce the effect of any external disturbances on the measurements, the tests are conducted in a closed box made from Plexiglass (PMMA) to provide optical access. This box is used in both the load cell and PIV experiments. Applied voltages of 8 to $16 \mathrm{kV}_{\mathrm{pp}}$ and carrier frequencies of 1 to $4 \mathrm{kHz}$ are tested. The waveform of the applied voltage is a sine. The thrust measurements are a part of an extensive work [25] which involves additional geometric variations of the actuator. An overview of the actuator and test parameters for all measurements is shown in table 1.

\subsection{Load cell}

For the direct thrust measurements an electronic ME-messsysteme KD-40S strain-gage load cell is used. The nominal force range is $\pm 2 \mathrm{~N}$ with an accuracy of $0.1 \%(2 \mathrm{mN})$. A light PMMA plate of $5 \mathrm{~mm}$ thickness, which carries the electrodes and dielectric, is directly mounted on the load cell. The connection of the electrodes to the HV and ground connectors is via thin copper wires to minimize signal contamination from external vibrations. Tests are repeated three times to minimize any error due to load-cell relaxation. The measurement is continued for $10 \mathrm{~s}$ before actuation and $30 \mathrm{~s}$ after in order to ensure that the steady flow regime is achieved. The thrust is then calculated by comparing the time-averaged readings from the two time periods.

\subsection{PIV setup}

Time-resolved PIV has been applied to characterize the flow field in the vicinity of the plasma actuator. This technique provides the required spatial and temporal resolution in order to characterize the transient behaviour of the thin wall jet induced by the actuator. A two component PIV configuration has been chosen since the large span of the actuator ensures minimal 3D effects. The two-dimensionality of the flow is also verified by hot-wire measurements.

The plasma actuator is placed flushed on the bottom of the Plexiglas box. A Photron Fastcam SA1 high-speed CCD camera of $1024 \times 1024$ pixels (full sensor size) is used to image the field-of-view (FOV). Image acquisition has been conducted at $10 \mathrm{KHz}$ rate in single-frame mode. This implies a time separation of $100 \mu$ s between successive images. A Nikkor $105 \mathrm{~mm}$ objective is set at f-stop 4 and is used along with extension tubes in order to achieve 0.8 magnification and a FOV of $15 \times 6 \mathrm{~mm}^{2}$. To achieve the high sample rate needed for these measurements, the sensor is cropped to a size of $1024 \times 512$ pixels. The air in the Plexiglas box is seeded with olive oil droplets of approximately $1 \mu \mathrm{m}$ diameter generated by a TSI atomizer. The particles at the mid-span of the actuator are illuminated by a light sheet of $2 \mathrm{~mm}$ thickness generated by a Quantronix Darwin-Duo laser system with an average output of $80 \mathrm{~W}$ at $3 \mathrm{kHz}$. The images are analysed using Davis 7.4 (Lavision $\mathrm{GmbH}$ ) by cross-correlating successive images. Final interrogation window size of $12 \times 12$ pixels and overlap factor of $75 \%$ are used. The interrogation windows are elongated in the wall normal direction using a $4: 1$ aspect ratio in order to obtain higher spatial resolution. The velocity vectors are returned on a grid of 13 vectors per $\mathrm{mm}$.

\section{Results}

\subsection{Load-cell thrust measurements}

The load-cell measurement offers a robust approach and has been applied to a large number of test cases. Detailed results can be found in [25]. In this paper the load-cell results are only presented for the voltage $(V)$ and frequency $(f)$ values identical to the one used for the PIV measurement.

The test cases studied in this paper are shown in table 1 . For voltage variation three cases with base frequency of 1 , 

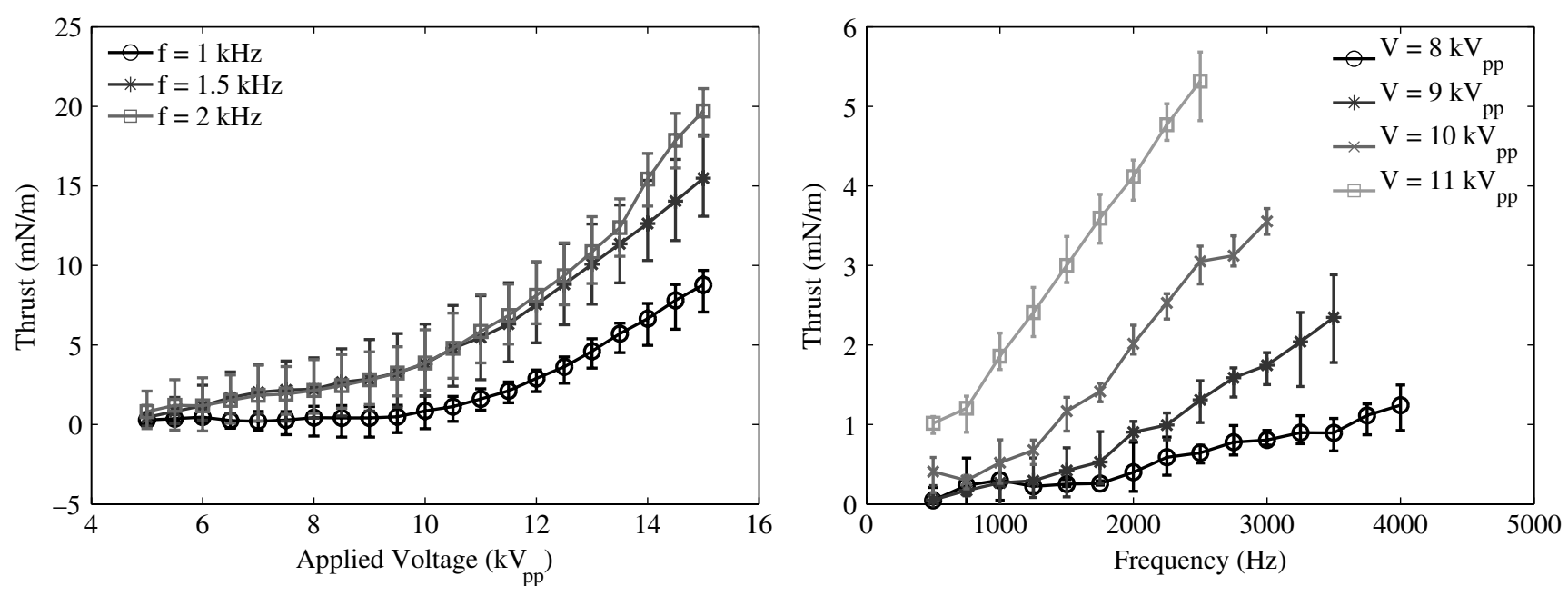

Figure 3. Thrust $\left(T_{x}\right)$ per length of actuator for different applied voltages and frequencies, as measured by the load cell.

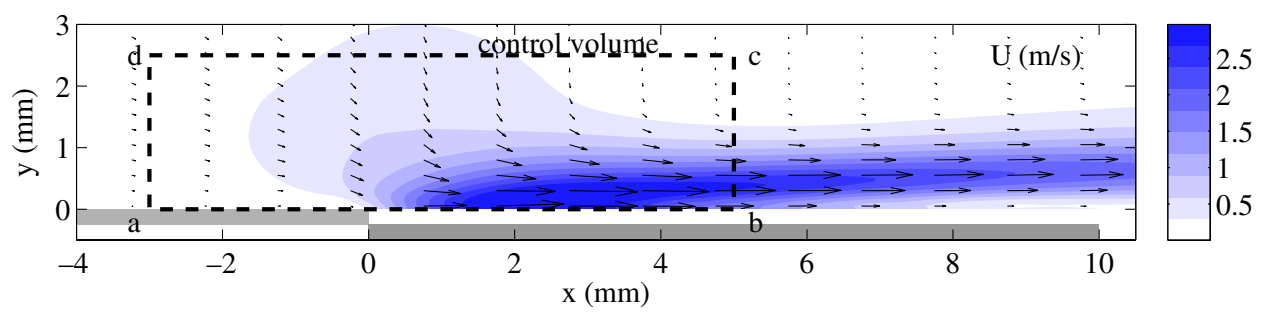

Figure 4. Time averaged velocity field and control volume for momentum balance $\left(12 \mathrm{kV}_{\mathrm{pp}}, 2 \mathrm{kHz}\right)$.

1.5 and $2 \mathrm{kHz}$ are done, while for frequency variation four cases with base voltage of $8,9,10$ and $11 \mathrm{kV}_{\mathrm{pp}}$ are performed. For the frequency variation the maximum tested frequency is limited by the slew rate of the $\mathrm{HV}$ amplifier $\left(350 \mathrm{~V} \mathrm{\mu s}^{-1}\right)$ and is reduced with increase in voltage amplitude. For the entire study, a sinusoidal waveform has been used for the HV signal.

Thrust results for voltage and frequency variation are presented in figure 3. Both voltage and frequency seem to influence the magnitude of thrust albeit in different manners. Thrust is related to voltage with a power law. Similar behaviour has been previously observed by Abe et al [21]. In contrast, the relation with frequency is linear.

\subsection{PIV measurements: estimation of thrust}

The dataset from the high-speed PIV measurement is used for the thrust estimation using the momentum balance technique. After the initial acceleration stage, the induced flow field develops into a wall jet. In the steady-state regime the momentum balance equations do not require evaluation of the acceleration terms. Steady state is reached in approximately $30 \mathrm{~ms}$ after actuation for the case of $8 \mathrm{kV}_{\mathrm{pp}}$. In this respect it is safe to average for approximately 400 frames spanning from $43 \mathrm{~ms}$ after the actuation to $83 \mathrm{~ms}$. Apart from the processing of the raw PIV data and time averaging, no other data processing is required.

The time-averaged flow field with the selected control volume is shown in figure 4 for the case of $12 \mathrm{kV}_{\mathrm{pp}}$. The momentum increase mainly occurs in the $x$-direction while a weak suction effect is observed upstream of the inner edge
Table 2. Section contribution in thrust $\left(\mathrm{mN} \mathrm{m}_{\mathrm{act}}^{-1}\right)$ for case of $12 \mathrm{kV}_{\mathrm{pp}}, 2 \mathrm{kHz}$.

\begin{tabular}{lrr}
\hline Section & \multicolumn{1}{l}{$T_{x}$} & \multicolumn{1}{l}{$T_{y}$} \\
\hline$d a$ & -0.131 & 0.0015 \\
$c d$ & 0.0160 & -0.1344 \\
$b c$ & 3.9828 & -0.0235 \\
Total & 3.8673 & -0.1564 \\
\hline
\end{tabular}

of the electrodes. The suction effect is an indication of a pressure gradient which is potentially strong in the vicinity of the actuator. As mentioned, any existing pressure gradient cannot be readily resolved using the time-averaged velocity data. It is therefore necessary to choose the control volume such that the pressure effects are minimized. The chosen control volume is a rectangular area spanning form $x=-3$ to $x=5 \mathrm{~mm}$ and $y=0$ to $y=2.5 \mathrm{~mm}$.

The momentum balance equation in the $x$ - and $y$-directions calculated using the terms in (8) and (9) reveals the significance of each boundary of the control volume. As is shown in table 2 and also apparent from the velocity field, the maximum contribution to thrust comes from the outflowing horizontal velocity from section $b c$. This implies that the very simple approach of calculating thrust from only one cross section of the jet would still deliver satisfying results. Nevertheless, since field data are available, the method used herein takes into account all sections of the control volume.

The thrust as a function of both applied voltage and frequency are shown in figure 5 . The same power law 

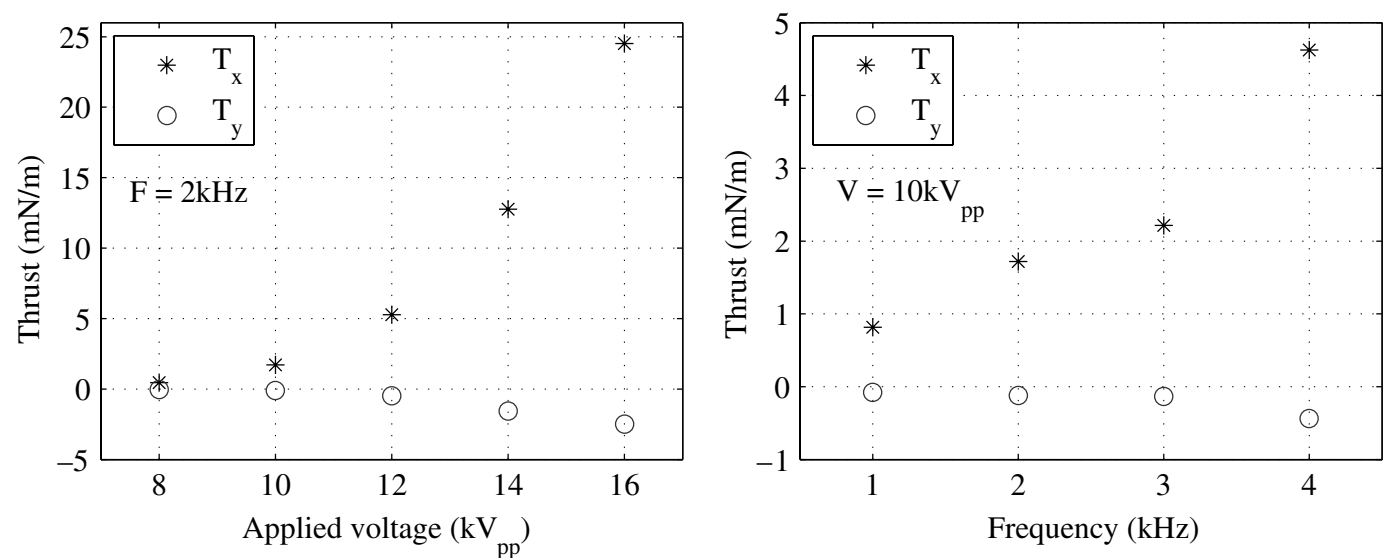

Figure 5. Thrust per length of actuator for different applied voltages and frequencies, as calculated by momentum balance on the PIV data.
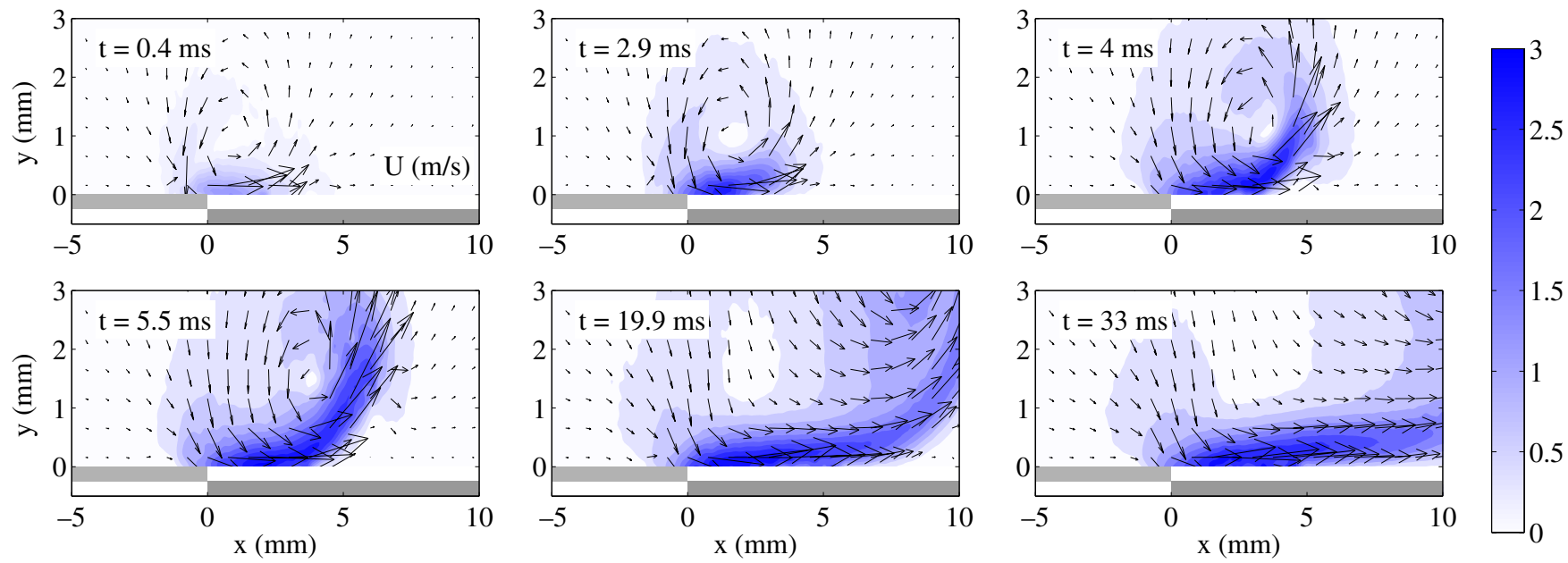

Figure 6. Temporal evolution of the total velocity field $(U)$ for the actuation case of $12 \mathrm{kV}_{\mathrm{pp}}, 2 \mathrm{kHz}$.

behaviour as observed in the direct thrust measurements is observed here for thrust in the $x$-direction with different voltages. A slight increase in thrust magnitude in the $y$-direction is observed with increase in the applied voltage. For different frequencies, the linear dependence of thrust appears to hold. A more concise comparison of the results from different techniques is performed at a subsequent section.

\subsection{PIV measurements: estimation of body force}

The two described approaches, reduced method and gradient method, are applied to the velocity field measured using PIV. Instantaneous snapshots of the evolving flowfield are presented in figure 6. The time values indicate elapsed time from the start of the actuation. The flow reacts immediately to the actuation with initial velocity components already starting to appear approximately $0.1 \mathrm{~ms}$ after the start of the actuation. The flow accelerates in a region starting from the end of the upper electrode and extending over half the covered electrode $(x=0$ to $5 \mathrm{~mm})$. The impulsive forcing on the flow creates a starting vortex which develops and moves out of the FOV in less than $40 \mathrm{~ms}$ after the start of the actuation. It is apparent that the general forcing of the actuator produces a wall jet which in turn creates a secondary weak suction effect upstream.
At this point, some specific features of the induced flow field should be discussed due to their effect on the results of the proposed technique. In a previous study [26], highfrequency velocity fluctuations are registered in the vicinity of the actuator. These fluctuations are a product of the carrier frequency at which the actuator is operating and reflect the underlying ion-neutral collision processes in the plasma region as reported in previous numerical studies [27]. These fluctuations appear in the PIV measurements of this work as well, corresponding to the carrier frequency for each case. More in-depth analysis of this phenomenon does not fall in the scope of this study. The proposed methods rely on the resolution of the accelerating flow and interpretation of that to a body force. In this process the first and second time derivatives of velocity are required. Due to the high-frequency fluctuations, these terms cannot be explicitly resolved without large artefacts. Based on the large difference between the time scale governing the acceleration of the flow and the time scale of these fluctuations one further assumption must be made. This states that although the flow, and to an extent the body force, fluctuates in time, the latter can be considered as quasisteady. This assumption can be justified by the fact that the rise time of the flow (accelerating part) is considerably larger than the fluctuation period. This is also a major assumption 


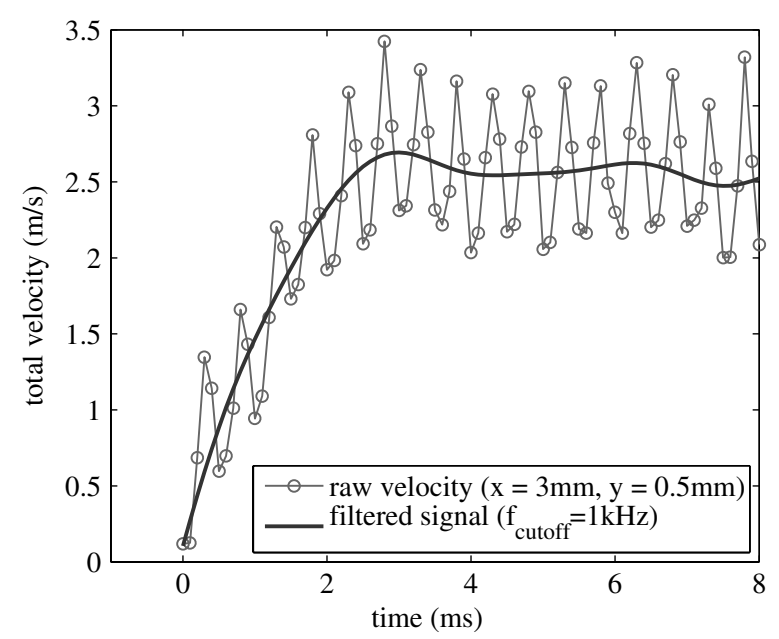

Figure 7. Raw and filtered signal of velocity in the developing flowfield.

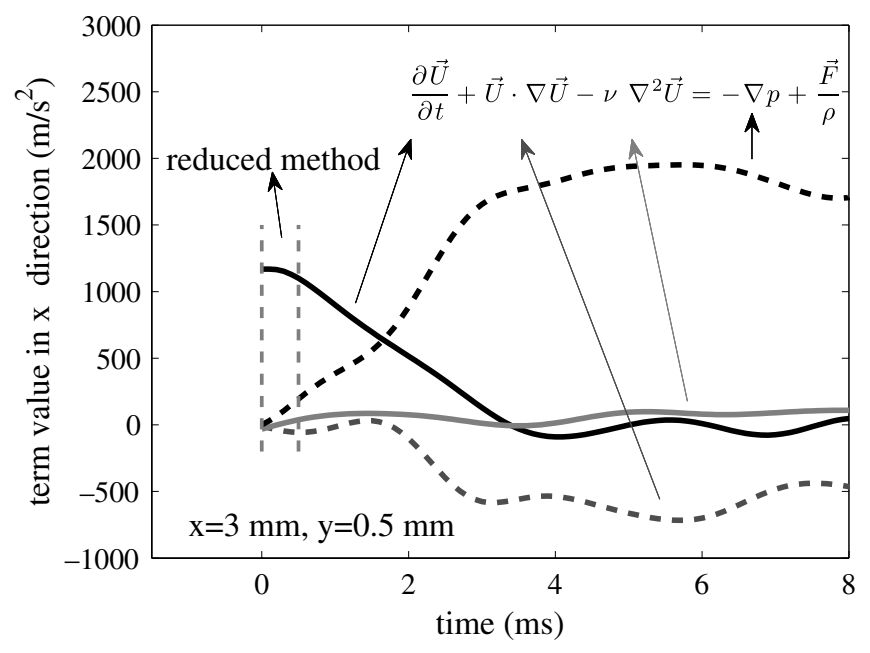

Figure 8. Temporal evolution of the NS terms governing the development of the flow in the $x$-direction $\left(12 \mathrm{kV}_{\mathrm{pp}}, 2 \mathrm{kHz}\right)$.

made in a large majority of first-principles modelling studies on the plasma operation. Based on this, the PIV time-series of the flow field snapshots is filtered in time with a sixth order low-pass Chebychev filter as shown in figure 7. The cutoff frequency is chosen based on the carrier frequency of the HV signal corresponding to the test case under investigation. For the majority of cases the cutoff frequency is half the carrier frequency. By filtering the high-frequency fluctuations, the development of the velocity field occurs smoothly and the time derivatives can be calculated with reasonable accuracy.

The reduced method involves the utilization of the very first moments (first $0.5 \mathrm{~ms}$ ) after actuation when all other terms apart from acceleration are negligible. The acceleration, convective, viscous and pressure gradient components of the NS are shown in figure 8 as a function of time after actuation $(t=0)$. The terms are calculated in the $x$-direction from the time-filtered PIV data for a given point located well into the expected force field. As anticipated, the acceleration term dominates the very first moments of actuation with a high and almost constant value. Convection and pressure gradient are initially negligible and eventually dominate the event while viscous effects have a small influence during the entire actuation period. It should be mentioned here that the pressure gradient term in figure 8 is calculated using the gradient method as discussed in section 2.1. The initial acceleration-dominant time span is visually confirmed to be $0.5 \mathrm{~ms}$ and provides reasonable results.

The spatial distribution of the body force, as calculated by the reduced method, is shown in figure 9 for the case of $12 \mathrm{kV}_{\mathrm{pp}}$ applied voltage and $2 \mathrm{kHz}$ carrier frequency. The PIV snapshots used for this case cover the first $0.5 \mathrm{~ms}$ after actuation and the force field is simply averaged over this span. The bulk of the force appears to be just downstream from the inner electrode edge while the horizontal extent spans almost a quarter of the covered electrode. In the wall normal direction the force field extends no more than $1 \mathrm{~mm}$ which confirms the very thin wall jet reported by this and numerous other investigations. The vertical force seems to be very weak compared with the horizontal component. Its major direction is towards the wall especially near the edge of the exposed electrode. For this case the cutoff frequency of the Chebychev filter is $1000 \mathrm{~Hz}$.

For the same case $\left(12 \mathrm{kV}_{\mathrm{pp}}, 2 \mathrm{kHz}\right)$, the spatial distribution of the body force using the gradient method is shown in figure 10. The first $20 \mathrm{~ms}$ after actuation are used and the force field is averaged over this time span. The general shape of the field is identical to the results from the reduced method. Nevertheless, the magnitude of the force attained with the gradient method is slightly larger than the reduced method's result. This small discrepancy can be attributed to modelling errors inherent in the eliminated terms of the reduced method as well as the ambiguity in selecting the initial acceleration time span. These facts support the higher accuracy of the gradient method.

One additional advantage of the gradient method over the reduced method is its relative independence on the time span used for the force calculation. In contrast to the reduced method there is no term elimination involved and no consideration should be made in the dominance of one term or another. Applying this method for the first $20 \mathrm{~ms}$ after actuation gives an almost steady in time body force. Several snapshots of the instantaneous total force field are shown in figure 11. The temporal coherence of the force distribution is an extra indication of the validity of the method as this was an important assumption made during the formulation of the technique.

The force distributions for different voltage and frequencies are presented in figure 12 and figure 13, respectively. For voltage variation the trend of increasing force with increased voltage holds. Of interest is the spatial extent of the force field along the dielectric. As the voltage increases, the active phase of the negative half cycle, where the electron avalanche occurs, increases in duration as reported in the excellent paper by Likhanskii et al [27]. This allows the plasma to propagate further downstream. Respectively, in the positive half cycle the corona regime is sustained for a longer period enhancing the movement of positive ions downstream. This is also verified by the force distributions for different frequencies. In this case, the spatial extent of the plasma 


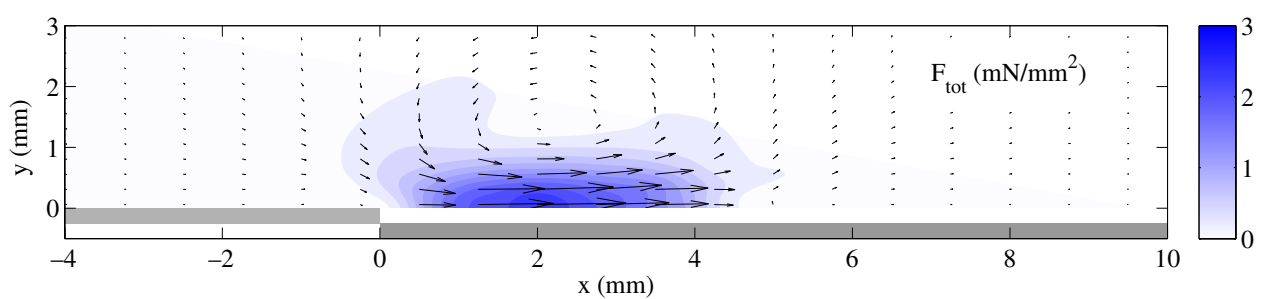

Figure 9. Spatial distribution and direction of the body force using the reduced method $\left(12 \mathrm{kV}_{\mathrm{pp}}, 2 \mathrm{kHz}\right)$.

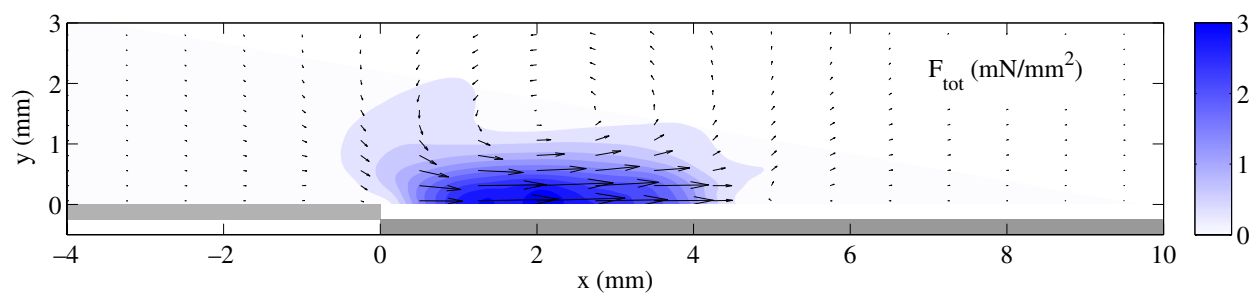

Figure 10. Spatial distribution of the body force using the gradient method $\left(12 \mathrm{kV}_{\mathrm{pp}}, 2 \mathrm{kHz}\right)$.
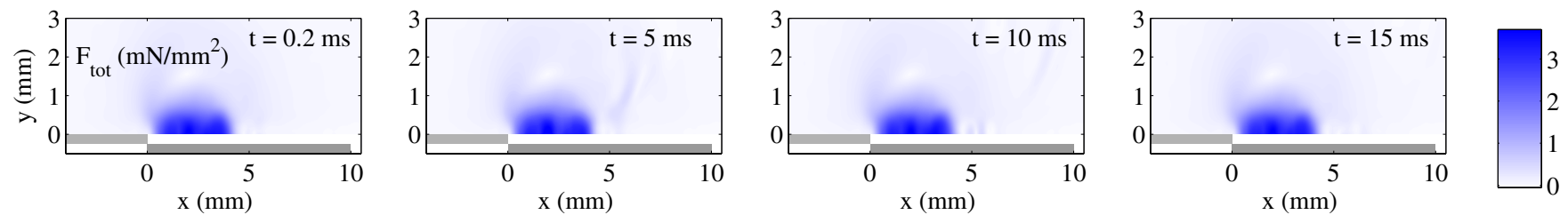

Figure 11. Instantaneous total force field obtained using the gradient method $\left(12 \mathrm{kV}_{\mathrm{pp}}, 2 \mathrm{kHz}\right)$.

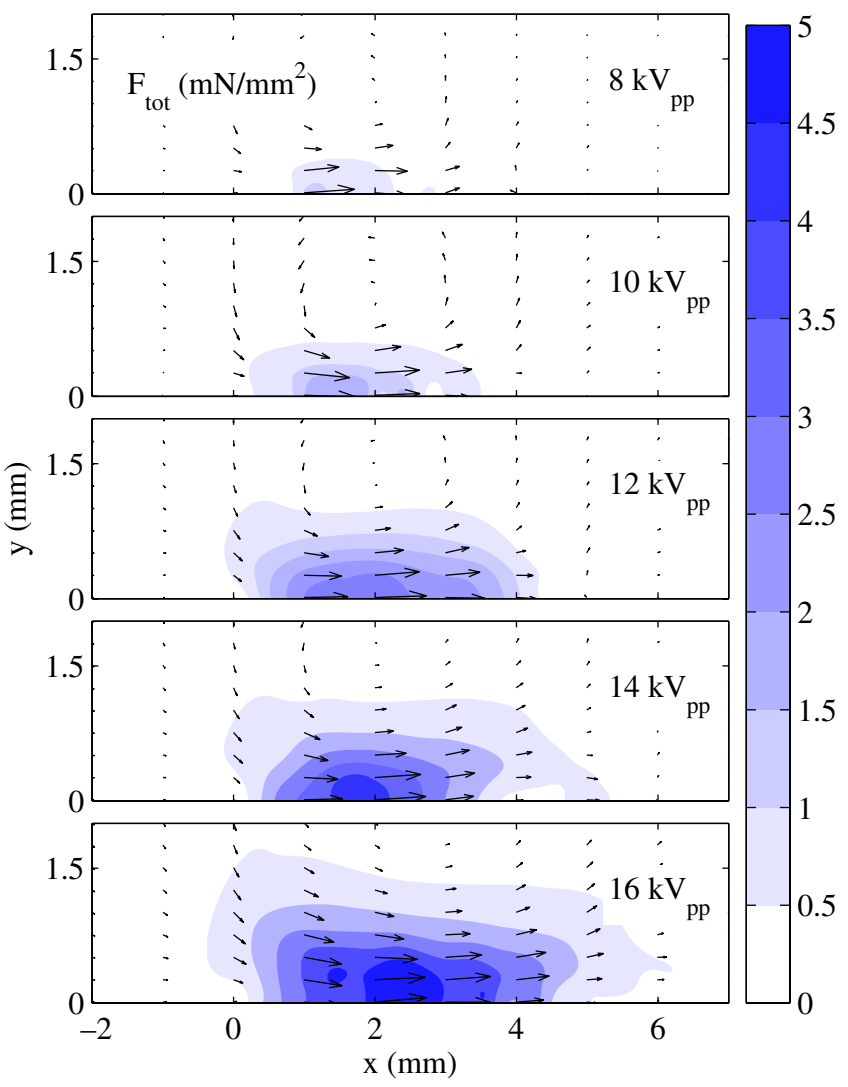

Figure 12. Body force distributions for different voltages as calculated by the gradient method $(f=2 \mathrm{kHz})$.

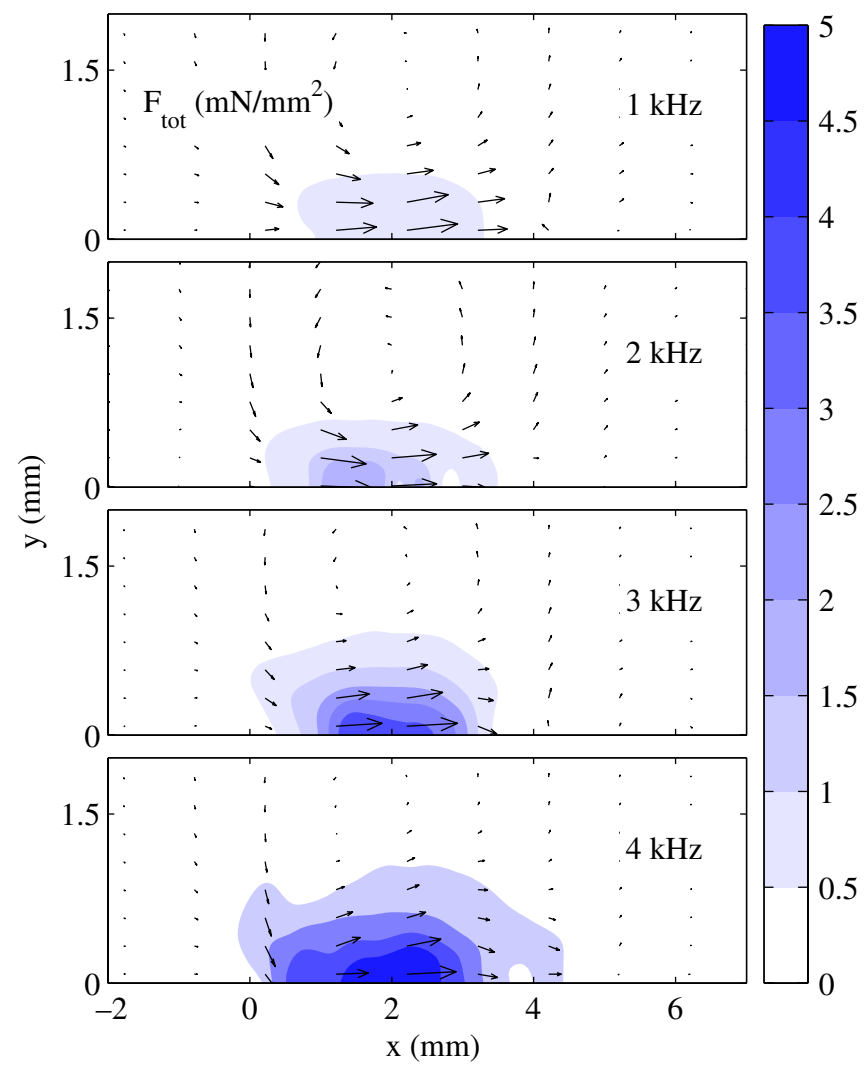

Figure 13. Body force distributions for different frequencies as calculated by the gradient method $\left(V=10 \mathrm{kV}_{\mathrm{pp}}\right)$. 
cloud remains almost the same as the applied voltage is fixed. The magnitude of the force, nevertheless, is increased since the momentum exchange between the ionized gas and neutral air is performed at a higher rate. Furthermore, a jump in maximum force also occurs from the case of $3 \mathrm{kHz}$ to the case of $4 \mathrm{kHz}$ as well as a secondary vertical force component towards the surface. This is an indication that $4-5 \mathrm{kHz}$ presents a threshold between two different regimes of operation. A low frequency regime exists (below $3-4 \mathrm{kHz}$ for these plasma scales), where the frequency is slow enough to allow full electron-ion recombination between the negative and positive half cycles. In contrast, for higher frequencies (above $4-5 \mathrm{kHz}$ ) the electron cloud remaining on the dielectric from the negative cycle is not completely destroyed before the positive cycle begins. The residual electrons are then pulled back to the exposed electrode causing further avalanche ionization as they move. As reported by Likhanskii, in this case the generated positive ions move towards and along the dielectric surface imparting momentum to the air. This might explain the secondary vertical force components located around $x=3 \mathrm{~mm}$ in the 3 and $4 \mathrm{kHz}$ cases. This is also mentioned by Soloviev and Krivtsov [28] where the discharge formation appears to be affected by the background particle concentration fields left from the previous HV cycles. Soloviev and Krivtsov suggest that the momentum input is dependent on the temporal overlap between two subsequent discharge cycles.

\subsection{Comparison}

An overall comparison between the thrust values attained by the proposed body force estimation methods and the two benchmark techniques is presented in figure 14 for different voltages and frequencies. It should be noted here that the thrust value from the proposed PIV techniques is calculated by integrating the body force value over the spatial domain.

For different voltages, a good agreement is observed between the different techniques. In the $x$-direction (horizontal body force) the momentum balance is located at the lower error margin of the load-cell measurements. The thrust estimated

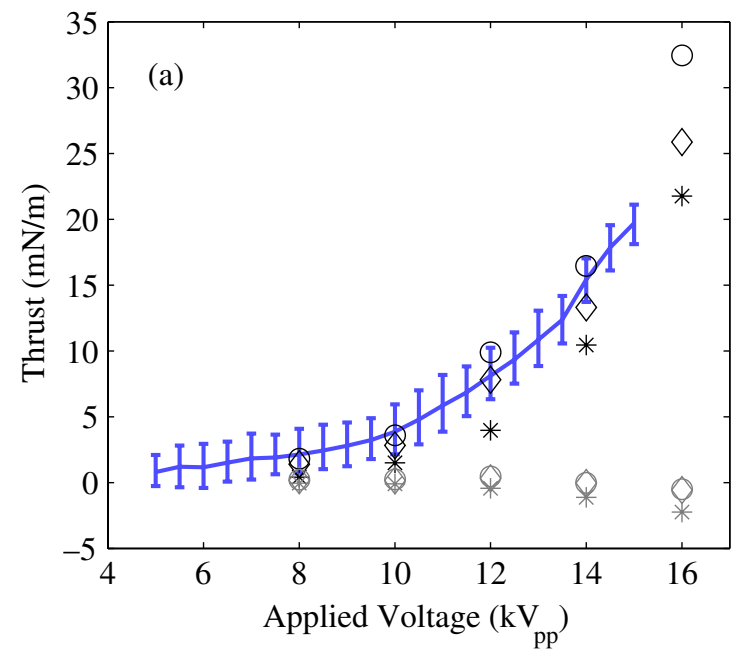

by the body force methods is systematically larger than the two other techniques although still in the error margin of the load-cell for the majority of cases. This is expected as both, the load cell measurements and the momentum balance calculations contain the shear stress at the wall. Additionally, the previously mentioned difference between the reduced and gradient methods is observed. This is attributed to the term elimination inherent in the reduced method.

For different frequencies, the absolute error stays in the same level while the relative error between the different methods is larger. This is partly due to the low applied voltage of $10 \mathrm{kV}_{\mathrm{pp}}$ that is used for this test series as the slew rate of the currently used $\mathrm{HV}$ amplifier is the limiting factor in increasing both voltage and frequency. The low voltage results in lower body force and the results are more susceptible to interference from external sources and other measurement errors. It is expected that the relative error would decrease with higher applied voltage.

\section{Conclusions}

A novel technique for the estimation of the body force field of plasma actuators is developed and tested. This involves the use of time-resolved PIV data on the evolving flowfield during actuator operation. The force amplitude and spatial distribution can be calculated through the estimation of the individual terms of the 2D incompressible NS equations and is conducted in two distinct ways. First, a dimensional analysis is performed on the temporal and spatial evolution of the velocity field. An initial period dominated by pure acceleration is identified and the latter is expanded explicitly in body force terms. Second, the full NS is estimated. The decoupling between the force and pressure gradient terms is performed based on the temporal stability of the body force and the initial zero pressure gradient.

The results from the tested methods are compared against well-established thrust measurement techniques and demonstrate sufficient agreement. Based on the comparison several comments can be made on the proposed

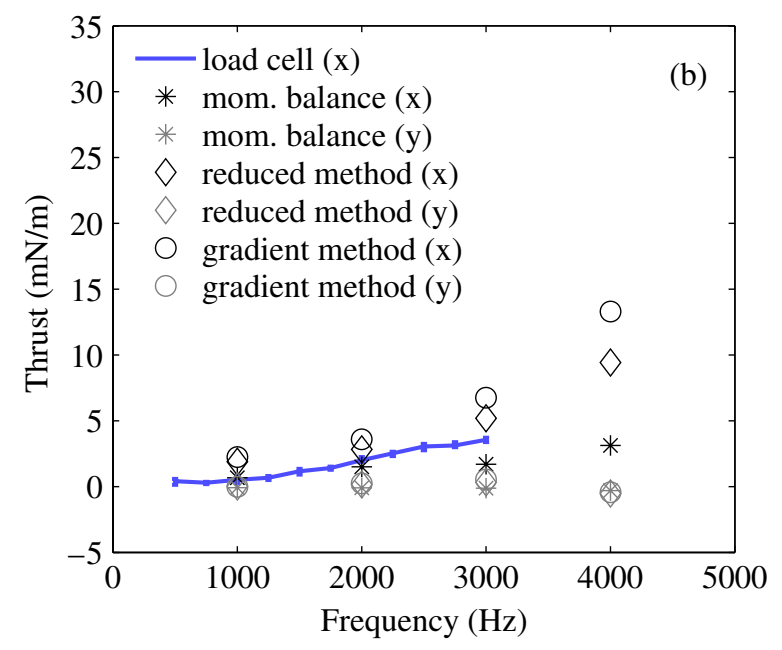

Figure 14. Thrust values from the tested methods as a function of applied voltage $(a)$ and frequency $(b)$. 
techniques. The reduced and gradient techniques produced consistently larger values of horizontal force than direct thrust measurements or momentum balance on the velocity data. This is mainly attributed to the inherent inclusion of the wall shear force, opposite the plasma body force, in the total thrust value obtained by the two benchmark techniques. The proposed techniques negate this effect since the calculation is done in the fluid volume not including the wall effects. Furthermore, a comparison can be made between the reduced and gradient methods. Although attractive because of its simplicity, the reduced method presents some drawbacks in respect to the gradient method. These are based on term elimination as well as on the semi-arbitrary choice of applied time length.

The general shape of the force field seems to be dictated by voltage and frequency for the current geometric configuration of the actuator. The shape resembles an elongated ellipse with the maximum force arising near the inner electrode edge. Increasing voltage has two major effects on the field being an increase in amplitude and an extension in the downstream direction over the covered electrode. This is attributed to the increase in the electric field and consequently the extension of the active phase for each of the two half cycles of the actuation signal. Compared with the voltage, the relation to frequency is slightly different since there is no visible change in shape but only in amplitude. A possible jump in operation regimes is visible for higher frequencies. A residual electron avalanche in the positive half cycle seems to occur for the cases of 3 and $4 \mathrm{kHz}$ although no definite conclusion can be made at this point.

\section{Acknowledgments}

This work is done within the framework of the CleanEra project at the TU Delft (www.cleanera.tudelft.nl). The authors would like to acknowledge Leo Molenwijk and Stefan Bernardy for their technical assistance.

\section{References}

[1] Post M L and Corke T C 2004 Separation control on high angle of attack airfoil using plasma actuators AIAA $J$. 42 2177-84

[2] Jukes T N, Choi K, Johnson G A and Scott S J 2006 Turbulent drag reduction by surface plasma through spanwise flow oscillation 3rd AIAA Flow Control Conf. (San Francisco, CA, June 2006) vol 3 pp 1687-700

[3] Jacob J D, Rivir R, Carter C and Estevadeordal J 2004 Boundary layer flow control using ac discharge plasma actuators 2nd AIAA Flow Control Conf. (Portland, OR, July 2004) number AIAA 2004-2128

[4] Huang J, Corke T C and Thomas F O 2006 Plasma actuators for separation control of low-pressure turbine blades AIAA J. 44 51-7

[5] Seraudie A, Aubert E, Naude N and Cambronne J P 2006 Effect of plasma actuators on a flat plate laminar boundary layer in subsonic conditions 3rd AIAA Flow Control Conf. (San Francisco, CA, June 2006) vol 2 pp 1065-73

[6] Li Y, Zhang X and Huang X 2010 The use of plasma actuators for bluff body broadband noise control Exp. Fluids 49 367-77
[7] Grundmann S and Tropea C 2008 Active cancellation of artificially introduced Tollmien-Schlichting waves using plasma actuators Exp. Fluids $\mathbf{4 4} 795-806$

[8] Moreau E 2007 Airflow control by non-thermal plasma actuators J. Phys. D: Appl. Phys. 40 605-36

[9] Corke T C, Enloe C L and Wilkinson S P 2010 Dielectric barrier discharge plasma actuators for flow control Annu. Rev. Fluid Mech. 42 505-29

[10] Likhanskii A V, Shneider M N, Opaits D F, Miles R B and Macheret S O 2007 Numerical modeling of dbd plasma actuators and the induced air flow 38th AIAA Plasmadynamics and Lasers Conf. (Miami, FL, June 2007) vol 2 pp 1060-72

[11] Jayaraman B, Cho Y and Shyy W 2007 Modeling of dielectric barrier discharge plasma actuator 38th AIAA Plasmadynamics and Lasers Conf. (Miami, FL, June 2007) vol 2 pp 1019-38

[12] Lagmich Y, Th Callegari, Pitchford L C and Boeuf J P 2008 Model description of surface dielectric barrier discharges for flow control J. Phys. D: Appl. Phys. 41095205

[13] Boeuf J P and Pitchford L C 2005 Electrohydrodynamic force and aerodynamic flow acceleration in surface dielectric barrier discharge J. Appl. Phys. 97103307

[14] Jayaraman B and Shyy W 2008 Modeling of dielectric barrier discharge-induced fluid dynamics and heat transfer Prog. Aerospace Sci. 44 139-91

[15] Singh K P, Roy S and Gaitonde D V 2006 Modeling of dielectric barrier discharge plasma actuator with atmospheric air chemistry 37th AIAA Plasmadynamics and Lasers Conf. (San Francisco, CA, June 2006) vol 2 pp 576-86

[16] Orlov D M, Apker T, He C, Othman H and Corke T C 2007 Modeling and experiment of leading edge separation control using sdbd plasma actuators 45th AIAA Aerospace Sciences Meeting and Exhibit (Reno, NV, January 2007) vol 15 pp 10651-68

[17] He C, Corke T C and Patel M P 2007 Numerical and experimental analysis of plasma flow control over a hump model 45th AIAA Aerospace Sciences Meeting and Exhibit (Reno, NV, January, 2007) vol 16 pp 11322-37

[18] Enloe C L, McHarg M G and McLaughlin T E 2008 Time-correlated force production measurements of the dielectric barrier discharge plasma aerodynamic actuator J. Appl. Phys. 103073302

[19] Forte M, Jolibois J, Pons J, Moreau E, Touchard G and Cazalens M 2007 Optimization of a dielectric barrier discharge actuator by stationary and non-stationary measurements of the induced flow velocity: application to airflow control Exp. Fluids 43 917-28

[20] Opaits D F, Shneider M N, Miles R B, Likhanskii A V and MacHeret S O 2008 Surface charge in dielectric barrier discharge plasma actuators Phys. Plasmas 15073505

[21] Abe T, Takizawa Y, Sato S and Kimura N 2008 Experimental study for momentum transfer in a dielectric barrier discharge plasma actuator AIAA J. 46 2248-56

[22] Dong B, Bauchire J M, Pouvesle J M, Magnier P and Hong D 2008 Experimental study of a DBD surface discharge for the active control of subsonic airflow J. Phys. D: Appl. Phys. 41155201

[23] Hoskinson A R, Hershkowitz and Ashpis D E 2008 Force measurements of single and double barrier dbd plasma actuators in quiescent air J. Phys. D: Appl. Phys. 41245209

[24] Anderson J D 2005 Fundamentals of Aerodynamics (New York: McGraw-Hill)

[25] Kotsonis M, Ghaemi S, Giepman R and Veldhuis L 2010 Experimental study on the body force field of dielectric 
barrier discharge actuators 41st AIAA Plasmadynamics and Laser Conf. (Chicago, IL, June 2010) number AIAA 2010-4630

[26] Benard N and Moreau E 2010 Capabilities of the dielectric barrier discharge plasma actuator for multi-frequency excitations J. Phys. D: Appl. Phys. 43145201
[27] Likhanskii A V, Shneider M N, MacHeret S O and Miles R B 2008 Modeling of dielectric barrier discharge plasma actuator in air J. Appl. Phys. 103053305

[28] Soloviev V R and Krivtsov V M 2009 Surface barrier discharge modelling for aerodynamic applications J. Phys. D: Appl. Phys. 42125208 